\title{
An Estimate of Goodness of Cubatures for the Unit Circle in $\mathbf{R}^{2}$
}

\author{
By J. I. Maeztu
}

\begin{abstract}
The Sarma-Eberlein estimate $s_{E}$ is an estimate of goodness of cubature formulae for $n$-cubes defined as the integral of the square of the formula truncation error, over a function space provided with a measure. In this paper, cubature formulae for the unit circle in $\mathbf{R}^{2}$ are considered and an estimate of the above type is constructed with the desirable property of being compatible with the symmetry group of the circle.
\end{abstract}

\section{Isometries and Two-dimensional Cubature Formulae. Let}

$$
S_{2}=\left\{(x, y) \in \mathbf{R}^{2}: x^{2}+y^{2} \leqslant 1\right\}
$$

be the unit circle in the two-dimensional Euclidean space $\mathbf{R}^{2}$ and let $\mathscr{U}\left(S_{2}\right)$ denote the symmetry group of $S_{2}$. This group consists of all linear bijective maps $u$ : $\mathbf{R}^{2} \rightarrow \mathbf{R}^{2}$ which preserve the Euclidean distance (that is, isometries of $\mathbf{R}^{2}$ leaving the origin invariant). Each element of $\mathscr{U}\left(S_{2}\right)$ can be identified with a $2 \times 2$ real orthogonal matrix and therefore

$$
\mathscr{U}\left(S_{2}\right)=\left\{u_{\alpha}, u_{\alpha} \circ v ; \alpha \in[0,2 \pi)\right\},
$$

where $u_{\alpha}$ denotes the rotation of $\alpha$ radians around the origin and $v$ is the reflection about any fixed straight line passing through the origin; thus

$$
\begin{gathered}
u_{\alpha}(x, y)=(x \cos \alpha-y \sin \alpha, x \sin \alpha+y \cos \alpha), \\
v(x, y)=(x,-y) .
\end{gathered}
$$

Let $w(x, y)$ be a normalized weight function compatible with $\mathscr{U}\left(S_{2}\right)$, that is, a real positive continuous function in the interior of $S_{2}$ such that

$$
\iint_{S_{2}} w(x, y) d x d y=1 \quad \text { and } \quad w \circ u=w \quad \text { for all } u \in \mathscr{U}\left(S_{2}\right)
$$

A cubature formula for the $w$-weighted circle $S_{2}$ has the form

$$
I(f)=Q_{N}(f)+E(f),
$$

Received May 14, 1985; revised November 27, 1985.

1980 Mathematics Subject Classification. Primary 65D32. 
where

$$
\begin{gathered}
I(f)=\iint_{S_{2}} w(x, y) f(x, y) d x d y, \\
Q_{N}(f)=\sum_{i=1}^{N} A_{i} f\left(x_{i}, y_{i}\right), \quad\left(x_{i}, y_{i}\right) \in S_{2},
\end{gathered}
$$

and the constants $A_{i}$ are independent of $f$.

Let us consider a symmetry $u \in \mathscr{U}\left(S_{2}\right)$ acting on (1.5). Since $I(f \circ u)=I(f)$, it leads to another cubature formula

$$
I(f)=Q_{N}^{\prime}(f)+E^{\prime}(f),
$$

where

$$
\begin{gathered}
Q_{N}^{\prime}(f)=Q_{N}(f \circ u)=\sum_{i=1}^{N} A_{i} f\left(u\left(x_{i}, y_{i}\right)\right), \\
E^{\prime}(f)=E(f \circ u) .
\end{gathered}
$$

Definition 1. For every $u \in \mathscr{U}\left(S_{2}\right)$, the cubature formulae (1.5) and (1.7) are said to be $\mathscr{U}\left(S_{2}\right)$-equivalent or equivalent with respect to the symmetry group of $S_{2}$.

The integration of a function on the $w$-weighted circle $S_{2}$ is independent of the pair of orthogonal axis $O X, O Y$ whose origin $O$ lies in the center of the circle. Therefore, all $\mathscr{U}\left(S_{2}\right)$-equivalent formulae have identical characteristics when they are considered as approximations of the operator $I$.

Therefore, any estimate of goodness for cubature formulae (1.5) should be compatible with the $\mathscr{U}\left(S_{2}\right)$-equivalence relation, that is, all $\mathscr{U}\left(S_{2}\right)$-equivalent formulae should have the same estimate of goodness. For instance, the degree of precision of a cubature formula (1.5) is an estimate compatible with $\mathscr{U}\left(S_{2}\right)$, because the space of polynomials of degree at most $n$ is invariant under all the symmetries in (1.2).

The aim of this paper is to construct an $\mathscr{U}\left(S_{2}\right)$-compatible estimate of goodness of cubature formulae for $S_{2}$ similar to that defined by V. L. N. Sarma in [3] for cubatures for the square.

The next section is devoted to recalling briefly some characteristics of the Sarma-Eberlein estimate that are useful for our purpose. A detailed exposition of the construction of this estimate can be found in [3], [4] and [5] and an excellent summary of these results in [6, pp. 188-192].

2. The Sarma-Eberlein Estimate of Goodness $s_{E}$. Let us consider the square

$$
C_{2}=\left\{(x, y) \in \mathbf{R}^{2}:|x| \leqslant 1,|y| \leqslant 1\right\}
$$

and cubature formulae

$$
I(f)=Q_{N}(f)+E(f),
$$

where

$$
\begin{gathered}
I(f)=\frac{1}{4} \iint_{C_{2}} f(x, y) d x d y \\
Q_{N}(f)=\sum_{i=1}^{N} A_{i} f\left(x_{i}, y_{i}\right), \quad\left(x_{i}, y_{i}\right) \in C_{2} .
\end{gathered}
$$


Sarma in [3], [4] defines the estimate of goodness of the cubature formula (2.1) as

$$
s_{E}^{2}=\int_{F S_{\infty}} E(f)^{2} d f
$$

where the integral is defined over the unit sphere of a normed space of functions provided with a measure defined as follows:

Let $l_{1}$ be the space of real sequences

$$
f=\left\{f_{n k} ; n=0,1, \ldots ; k=0,1, \ldots, n\right\}
$$

such that

$$
\|f\|_{1}=\sum_{n, k}\left|f_{n k}\right|<\infty ; \quad n=0,1, \ldots ; k=0,1, \ldots, n .
$$

The unit sphere $S_{\infty}=\left\{f \in l_{1}:\|f\|_{1} \leqslant 1\right\}$ is compact in the weak*-topology of $l_{1}$, and an elementary integral defined for the weak*-continuous real functions on $S_{\infty}$ can be extended by the Daniell process inducing a countably additive measure on $S_{\infty}$

Among the properties of this measure, let us recall that

$$
\begin{gathered}
\int_{S_{\infty}} f_{n k} f_{m l} d f=0 \quad \text { if }(n, k) \neq(m, l), \\
\int_{S_{\infty}} f_{n k}^{2} d f=\frac{2^{n+2}}{(n+2) !(n+3) !}=q_{n}^{2} .
\end{gathered}
$$

Real two-dimensional power series

$$
f(x, y)=\sum_{n, k} f_{n k} x^{n-k} y^{k} ; \quad n=0,1, \ldots ; k=0,1, \ldots, n,
$$

whose coefficients satisfy the condition

$$
\|f\|_{1}=\sum_{n, k}\left|f_{n k}\right|<\infty
$$

converge uniformly and absolutely for all points $(x, y) \in C_{2}$.

The space $F l_{1}$ of all functions defined by (2.8) and (2.9) can be identified with the sequence space $l_{1}$ and is dense in the space $\mathscr{C}\left(C_{2}\right)$ of all real continuous functions on $C_{2}$ with the uniform norm. This identification allows us to consider the above integral as an integral over the unit sphere $F S_{\infty}$ of the function space $F l_{1}$.

The truncation error $E(f)$ of the cubature formula (2.1) is a continuous linear form over $\mathscr{C}\left(C_{2}\right)$ with the uniform norm and therefore also over $F l_{1}$ with the $\|\cdot\|_{1}$-norm. Using (2.6) and (2.7), it follows that the estimate $s_{E}$ defined by (2.3) can be written as

$$
s_{E}^{2}=\sum_{n=0}^{\infty} q_{n}^{2} e_{n}^{2},
$$

where $q_{n}$ is defined in (2.7) and

$$
e_{n}^{2}=\sum_{k=0}^{n} E\left(x^{n-k} y^{k}\right)^{2}
$$

It should be noted that the identification of $l_{1}$ and $F l_{1}$ is made through the monomials $x^{n-k} y^{k}$ and the use of these particular functions makes $s_{E}$ compatible with $\mathscr{U}\left(C_{2}\right)$, the symmetry group of $C_{2}$, in the sense described in the previous 
section. In effect, $\mathscr{U}\left(C_{2}\right)$ consists of the eight symmetries

$$
(x, y) \rightarrow( \pm x, \pm y) ; \quad(x, y) \rightarrow( \pm y, \pm x)
$$

and the equalities

$$
\begin{aligned}
e_{n}^{2} & =\sum_{k=0}^{n} E\left(x^{n-k} y^{k}\right)^{2}=\sum_{k=0}^{n} E\left(( \pm x)^{n-k}( \pm y)^{k}\right)^{2} \\
& =\sum_{k=0}^{n} E\left(( \pm y)^{n-k}( \pm x)^{k}\right)^{2}
\end{aligned}
$$

imply that $\mathscr{U}\left(C_{2}\right)$-equivalent cubature formulae have the same estimate of goodness $s_{E}$. Unfortunately, this estimate of goodness is not useful for cubature formulae (1.5), (1.6) for the unit circle $S_{2}$, because it is not compatible with $\mathscr{U}\left(S_{2}\right)$, as can be computationally checked. For instance, taking $w(x, y)=1 / \pi$, the cubature formula (degree 3, 4 points) given by

$$
Q_{4}(f)=\frac{1}{4}[f(\sqrt{2} / 2,0)+f(-\sqrt{2} / 2,0)+f(0, \sqrt{2} / 2)+f(0,-\sqrt{2} / 2)]
$$

has an estimate of goodness $s_{E}=(-4) 1.75032$, whereas the $\mathscr{U}\left(S_{2}\right)$-equivalent formula (use a rotation of $\pi / 4$ radians) given by

$$
\begin{aligned}
Q_{4}(f)= & \frac{1}{4}[f(1 / 2,1 / 2)+f(-1 / 2,1 / 2) \\
& +f(1 / 2,-1 / 2)+f(-1 / 2,-1 / 2)]
\end{aligned}
$$

has an estimate of goodness $s_{E}=(-4) 3.81547$.

3. An Estimate of Goodness of Cubatures for the Unit Circle. In the previous section, the sequence space $l_{1}$ was identified with the space of functions $F l_{1}$ by using the family of monomials $\left\{x^{n-k} y^{k} ; n=0,1, \ldots ; k=0,1, \ldots, n\right\}$, but we can also identify $l_{1}$ with other subspaces of $\mathscr{C}\left(C_{2}\right)$ or $\mathscr{C}\left(S_{2}\right)$ by using other families of polynomials. For each $n$, let us denote

$$
M_{n}=\left\{a_{0} x^{n}+a_{1} x^{n-1} y+\cdots+a_{n} y^{n} ; a_{i} \in \mathbf{R}\right\}
$$

and let

$$
\Phi_{n}=\left\{\varphi_{n 0}, \ldots, \varphi_{n n}\right\} \subset M_{n}
$$

be a basis of $M_{n}$, i.e., $M_{n}=\operatorname{span} \Phi_{n}$.

If the polynomials $\varphi_{n k}$ satisfy

$$
\left\|\varphi_{n k}\right\|_{\infty}=\max _{(x, y) \in S_{2}}\left|\varphi_{n k}(x, y)\right| \leqslant c ; \quad n=0,1, \ldots ; k=0,1, \ldots, n,
$$

then the series

$$
f(x, y)=\sum_{n, k} f_{n k} \varphi_{n k}(x, y)
$$

whose coefficients satisfy (2.9) converge uniformly and absolutely for all points $(x, y) \in S_{2}$. If we denote $\Phi=\left\{\Phi_{1}, \Phi_{2}, \ldots\right\}$, the space $F l_{1}(\Phi)$ of all functions defined by (3.4) and (2.9) can be identified with the sequence space $l_{1}$. Let us note that $F l_{1}(\Phi)$ contains all real polynomials in two variables and therefore is dense in $\mathscr{C}\left(S_{2}\right)$ with the uniform norm.

This identification allows us to define, in a natural way, an estimate of goodness for cubatures (1.5) by

$$
s_{E}^{2}(\Phi)=\int_{F S_{\infty}(\Phi)} E(f)^{2} d f
$$


where

$$
F S_{\infty}(\Phi)=\left\{f \in F l_{1}(\Phi): \sum_{n, k}\left|f_{n k}\right| \leqslant 1\right\} .
$$

It is straightforward to deduce that this estimate can be expressed by

$$
s_{E}^{2}(\Phi)=\sum_{n=0}^{\infty} q_{n}^{2} e_{n}^{2}\left(\Phi_{n}\right)
$$

where $q_{n}^{2}$ is given in (2.7) and

$$
e_{n}^{2}\left(\Phi_{n}\right)=\sum_{k=0}^{n} E\left(\varphi_{n k}\right)^{2}
$$

Our problem at this stage is to choose suitable families $\Phi_{n}$ satisfying (3.3), such that the estimate $s_{E}^{2}(\Phi)$ is compatible with the symmetry group $\mathscr{U}\left(S_{2}\right)$ in the sense described in Section 1.

As the matrix

$$
\left(\begin{array}{cc}
\cos \alpha, & -\sin \alpha \\
\sin \alpha, & \cos \alpha
\end{array}\right)
$$

associated with the rotation $u_{\alpha} \in \mathscr{U}\left(S_{2}\right)$ has eigenvalues $e^{i \alpha}, e^{-i \alpha}$ and eigenvectors $(1, i)^{T},(1,-i)^{T}$, the use of complex arithmetic will simplify the calculations. Let us denote

$$
M_{n}^{*}=\left\{a_{0} x^{n}+a_{1} x^{n-1} y+\cdots+a_{n} y^{n} ; a_{i} \in \mathbf{C}\right\}
$$

and let

$$
\Phi_{n}^{*}=\left\{\varphi_{n 0}^{*}, \ldots, \varphi_{n n}^{*}\right\}
$$

be a basis of $M_{n}^{*}$, i.e., $M_{n}^{*}=\operatorname{span} *\left(\Phi_{n}^{*}\right)$.

Considering the natural complexification of linear operators

$$
E(f+i g)=E(f)+i E(g)
$$

with the standard complex notation

$$
|E(f+i g)|^{2}=\overline{E(f+i g)} E(f+i g)=E(f)^{2}+E(g)^{2},
$$

we can define

$$
e_{n}^{2}\left(\Phi_{n}^{*}\right)=\sum_{k=0}^{n}\left|E\left(\varphi_{n k}^{*}\right)\right|^{2}
$$

THEOREM 1. For every $n$, let $\Phi_{n}^{*}=\left\{\varphi_{n 0}^{*}, \ldots, \varphi_{n n}^{*}\right\}$ and $\Phi_{n}=\left\{\varphi_{n 0}, \ldots, \varphi_{n n}\right\}$ be bases of $M_{n}^{*}$ and $M_{n}$, respectively, satisfying

(i) $\left(\varphi_{n 0}, \ldots, \varphi_{n n}\right)^{T}=A_{n}\left(\varphi_{n 0}^{*}, \ldots, \varphi_{n n}^{*}\right)^{T}$ where $A_{n}$ is an $n \times n$ complex unitary matrix, i.e., $A^{H}=A^{-1}$;

(ii) $\sum_{k=0}^{n}\left|E\left(\varphi_{n k}^{*}\right)\right|^{2}=\sum_{k=0}^{n}\left|E\left(\varphi_{n k}^{*} \circ u_{\alpha}\right)\right|^{2}=\sum_{k=0}^{n}\left|E\left(\varphi_{n k}^{*} \circ u_{\alpha} \circ v\right)\right|^{2}$ for all $\alpha \in$ $[0,2 \pi)$;

(iii) there exists a $c \in \mathbf{R}$ such that $\left\|\varphi_{n k}\right\|_{\infty} \leqslant c$ for all $n, k$.

Then, the estimate $s_{E}(\Phi)$ associated with the family $\Phi=\left\{\Phi_{0}, \Phi_{1}, \ldots\right\}$ is compatible with the symmetry group $\mathscr{U}\left(S_{2}\right)$. 
Proof. Let us remark that the operators

$$
\begin{aligned}
& f^{*} \in M_{n}^{*} \rightarrow E\left(f^{*}\right) \in \mathbf{C}, \\
& f^{*} \in M_{n}^{*} \rightarrow f^{*} \circ u_{\alpha} \in M_{n}^{*}, \\
& f^{*} \in M_{n}^{*} \rightarrow f^{*} \circ u_{\alpha} \circ v \in M_{n}^{*}
\end{aligned}
$$

are linear and therefore "pass through the matrix $A_{n}$ ".

Moreover, $E\left(\varphi_{n k}\right)$ and $E\left(\varphi_{n k} \circ u\right)$ are real and therefore

$$
\begin{aligned}
\sum_{k=0}^{n} E & \left(\varphi_{n k} \circ u_{\alpha}\right)^{2} \\
& =\left(\overline{E\left(\varphi_{n 0} \circ u_{\alpha}\right)}, \ldots, \overline{E\left(\varphi_{n n} \circ u_{\alpha}\right)}\right)\left(E\left(\varphi_{n 0} \circ u_{\alpha}\right), \ldots, E\left(\varphi_{n n} \circ u_{\alpha}\right)\right)^{T} \\
& =\left(\overline{E\left(\varphi_{n 0}^{*} \circ u_{\alpha}\right)}, \ldots, \overline{E\left(\varphi_{n n}^{*} \circ u_{\alpha}\right)}\right) A_{n}^{H} A_{n}\left(E\left(\varphi_{n 0}^{*} \circ u_{\alpha}\right), \ldots, E\left(\varphi_{n n}^{*} \circ u_{\alpha}\right)\right)^{T} \\
& =\sum_{k=0}^{n}\left|E\left(\varphi_{n k}^{*} \circ u_{\alpha}\right)\right|^{2}=\sum_{k=0}^{n}\left|E\left(\varphi_{n k}^{*}\right)\right|^{2} \\
& =\left(\overline{E\left(\varphi_{n 0}^{*}\right)}, \ldots, \overline{E\left(\varphi_{n n}^{*}\right)}\right)\left(E\left(\varphi_{n 0}^{*}\right), \ldots, E\left(\varphi_{n n}^{*}\right)\right)^{T} \\
& =\left(\overline{E\left(\varphi_{n 0}\right)}, \ldots, \overline{E\left(\varphi_{n n}\right)}\right) A_{n} A_{n}^{H}\left(E\left(\varphi_{n 0}\right), \ldots, E\left(\varphi_{n n}\right)\right)^{T}=\sum_{k=0}^{n} E\left(\varphi_{n k}\right)^{2}
\end{aligned}
$$

given that $A_{n}$ is unitary. Similarly, it can be shown that

$$
\sum_{k=0}^{n} E\left(\varphi_{n k} \circ u_{\alpha} \circ v\right)^{2}=\sum_{k=0}^{n} E\left(\varphi_{n k}\right)^{2},
$$

and therefore it follows in a straightforward way that $s_{E}(\Phi)$ is compatible with $\mathscr{U}\left(S_{2}\right)$.

Now let us consider the complex polynomials

$$
\varphi_{n k}^{*}=(x+i y)^{n-k}(x-i y)^{k} \in M_{n}^{*}
$$

obtained from the monomials $x^{n-k} y^{k}$ by a linear transformation with Jacobian

$$
J=\left|\begin{array}{cc}
1 & i \\
1 & -i
\end{array}\right|=-2 i
$$

so that $\varphi_{n 0}^{*}, \ldots, \varphi_{n n}^{*}$ are linearly independent in $M_{n}^{*}$.

Also,

$$
\begin{aligned}
& \left(\varphi_{n k}^{*} \circ u_{\alpha}\right)(x, y)=\varphi_{n k}^{*}(x \cos \alpha-y \sin \alpha, x \sin \alpha+y \cos \alpha) \\
& \quad=e^{i(n-k) \alpha}(x+i y)^{n-k} e^{-i k \alpha}(x-i y)^{k}=e^{i(n-2 k) \alpha} \varphi_{n k}^{*}(x, y),
\end{aligned}
$$

thus

$$
\sum_{k=0}^{n}\left|E\left(\varphi_{n k}^{*} \circ u_{\alpha}\right)\right|^{2}=\sum_{k=0}^{n}\left|E\left(\varphi_{n k}^{*}\right)\right|^{2}
$$

Similarly,

$$
\begin{aligned}
\left(\varphi_{n k}^{*} \circ u_{\alpha} \circ v\right)(x, y) & =\left(\varphi_{n k}^{*} \circ u_{\alpha}\right)(x,-y)=e^{i(n-2 k) \alpha}(x-i y)^{n-k}(x+i y)^{k} \\
& =e^{i(n-2 k) \alpha} \varphi_{n, n-k}^{*}(x, y),
\end{aligned}
$$


and then

$$
\sum_{k=0}^{n}\left|E\left(\varphi_{n k}^{*} \circ u_{\alpha} \circ v\right)\right|^{2}=\sum_{k=0}^{n}\left|E\left(\varphi_{n k}^{*}\right)\right|^{2}
$$

Therefore, for each $n$, the family $\Phi_{n}^{*}=\left\{\varphi_{n 0}^{*}, \ldots, \varphi_{n n}^{*}\right\}$ is a basis of $M_{n}^{*}$ which satisfies the hypothesis (ii) of Theorem 1.

For $k<n / 2$ let us define

$$
\begin{aligned}
\varphi_{n k} & =\frac{1}{\sqrt{2}}\left(\varphi_{n k}^{*}+\varphi_{n, n-k}^{*}\right) \\
& =\frac{1}{\sqrt{2}}\left(x^{2}+y^{2}\right)^{k}\left[(x+i y)^{n-2 k}+(x-i y)^{n-2 k}\right], \\
\varphi_{n, n-k} & =\frac{1}{\sqrt{2} i}\left(\varphi_{n k}^{*}-\varphi_{n, n-k}^{*}\right) \\
& =\frac{1}{\sqrt{2} i}\left(x^{2}+y^{2}\right)^{k}\left[(x+i y)^{n-2 k}-(x-i y)^{n-2 k}\right],
\end{aligned}
$$

and if $n$ is even,

$$
\varphi_{n, n / 2}=\varphi_{n, n / 2}^{*}=\left(x^{2}+y^{2}\right)^{n / 2} \text {. }
$$

Then, $\Phi_{n}=\left\{\varphi_{n 0}, \ldots, \varphi_{n n}\right\}$ is formed by polynomials with real coefficients and is a basis of $M_{n}$. Also the matrix $A_{n}$ of Theorem 1 that relates the elements of $\Phi_{n}$ and $\Phi_{n}^{*}$ is a unitary matrix, because the matrices

$$
\left(\begin{array}{ll}
\frac{1}{\sqrt{2}}, & \frac{1}{\sqrt{2}} \\
\frac{1}{\sqrt{2} i}, & \frac{-1}{\sqrt{2} i}
\end{array}\right)
$$

that relate the pairs $\left(\varphi_{n k}, \varphi_{n, n-k}\right)$ and $\left(\varphi_{n k}^{*}, \varphi_{n, n-k}^{*}\right)$ are unitary. Moreover, it can easily be shown that

$$
\left\|\varphi_{n k}\right\|_{\infty}=\left\|\varphi_{n, n-k}\right\|_{\infty}=\sqrt{2}, \quad k<n / 2
$$

and $\left\|\varphi_{n, n / 2}\right\|_{\infty}=1$ for $n$ even.

Using the results above, and applying Theorem 1, we deduce the following

THEOREM 2. Let $\Phi=\left\{\Phi_{0}, \Phi_{1}, \ldots\right\}$ where, for each $n, \Phi_{n}=\left\{\varphi_{n 0}, \ldots, \varphi_{n n}\right\}$ is the basis of $M_{n}$ defined by (3.18), (3.19) and (3.20). Then the estimate $s_{E}(\Phi)$ defined by (3.5) is an estimate of goodness of cubature formulae for the unit circle that is compatible with the symmetry group $\mathscr{U}\left(S_{2}\right)$.

Following the proof of Theorem 1, we can also deduce that

$$
s_{E}^{2}(\Phi)=\sum_{n=0}^{\infty} q_{n}^{2} \sum_{k=0}^{n} E\left(\varphi_{n k}\right)^{2}=\sum_{n=0}^{\infty} q_{n}^{2} \sum_{k=0}^{n}\left|E\left(\varphi_{n k}^{*}\right)\right|^{2},
$$

and therefore the estimate $s_{E}(\Phi)$ can be calculated using any of these two expressions. 
TABLE 1

\begin{tabular}{|l|r|r|c|}
\hline Formula & $\mathrm{D}$ & $\mathrm{N}$ & $s_{E}(\Phi)$ \\
\hline Centroid & 1 & 1 & $(-2) 3.72941$ \\
$S_{2}: 3-1$ & 3 & 4 & $(-3) 1.52574$ \\
$S_{2}: 5-1$ & 5 & 7 & $(-5) 4.17361$ \\
$S_{2}: 5-2$ & 5 & 9 & $(-5) 1.56155$ \\
$S_{2}: 7-1$ & 7 & 12 & $(-7) 7.32827$ \\
$S_{2}: 7-2$ & 7 & 16 & $(-7) 7.31334$ \\
$S_{2}: 9-1$ & 9 & 19 & $(-10) 2.86050$ \\
$S_{2}: 9-3$ & 9 & 21 & $(-9) 8.64763$ \\
$S_{2}: 9-5$ & 9 & 28 & $(-10) 5.70093$ \\
$S_{2}: 11-1$ & 11 & 28 & $(-11) 7.64307$ \\
$S_{2}: 11-2$ & 11 & 28 & $(-12) 2.00147$ \\
$S_{2}: 11-3$ & 11 & 28 & $(-11) 4.55280$ \\
$S_{2}: 11-4$ & 11 & 32 & $(-11) 7.64002$ \\
$S_{2}: 13-1$ & 13 & 37 & $(-14) 3.05146$ \\
$S_{2}: 13-2$ & 13 & 41 & $(-14) 1.03972$ \\
$S_{2}: 15-1$ & 15 & 44 & $(-15) 2.92306$ \\
$S_{2}: 15-2$ & 15 & 48 & $(-15) 2.88250$ \\
$S_{2}: 17-1$ & 17 & 61 & $(-20) 4.97655$ \\
\hline
\end{tabular}

Table 1 shows the values of $s_{E}(\Phi)$ for some cubature formulae (1.5) for the unit circle with $w(x, y)=1 / \pi$. The nomenclature of these formulae corresponds to the one in [6, pp. 277-289]. $\mathrm{N}$ stands for the number of nodes and $\mathrm{D}$ for the degree of precision.

Departamento de Matematica Aplicada

Facultad de Ciencias

Apartado 644

Bilbao, Spain

1. H. S. M. CoXeTER, Introduction to Geometry, Wiley, New York, 1969.

2. D. J. S. Robinson, A Course in the Theory of Groups, Springer-Verlag, Berlin and New York, 1982.

3. V. L. N. SARMA, “A generalisation of Eberlein's integral over function space," Trans. Amer. Math. Soc., v. 121, 1966, pp. 52-61.

4. V. L. N. SARMA, "Eberlein measure and mechanical quadrature formulae I. Basic theory," Math. Comp., v. 22, 1968, pp. 607-616.

5. V. L. N. SaRma \& A. H. Stroud, "Eberlein measure and mechanical quadrature formulae II. Numerical results," Math. Comp., v. 23, 1969, pp. 781-784.

6. A. H. STroud, Approximate Calculation of Multiple Integrals, Prentice-Hall, Englewood Cliffs, N. J., 1971. 